\title{
RESEARCH
}

Open Access

\section{Unmasking viral sequences by metagenomic next-generation sequencing in adult human blood samples during steroid-refractory/dependent graft-versus- host disease}

M. C. Zanella ${ }^{1,2^{*}}$ (D, S. Cordey ${ }^{2,3}$, F. Laubscher ${ }^{2,3}$, M. Docquier ${ }^{4,5}$, G. Vieille ${ }^{2}$, C. Van Delden ${ }^{1,3}$, V. Braunersreuther $^{6}$, Mc Kee TA ${ }^{6}$, J. A. Lobrinus ${ }^{6}$, S. Masouridi-Levrat ${ }^{3,7}$, Y. Chalandon ${ }^{3,7}$, L. Kaiser ${ }^{1,2,3,8+}$ and D. L. Vu $u^{1,2,3+}$

\begin{abstract}
Background: Viral infections are common complications following allogeneic hematopoietic stem cell transplantation (allo-HSCT). Allo-HSCT recipients with steroid-refractory/dependent graft-versus-host disease (GvHD) are highly immunosuppressed and are more vulnerable to infections with weakly pathogenic or commensal viruses. Here, twenty-five adult allo-HSCT recipients from 2016 to 2019 with acute or chronic steroid-refractory/dependent GvHD were enrolled in a prospective cohort at Geneva University Hospitals. We performed metagenomics next-generation sequencing (mNGS) analysis using a validated pipeline and de novo analysis on pooled routine plasma samples collected throughout the period of intensive steroid treatment or second-line GvHD therapy to identify weakly pathogenic, commensal, and unexpected viruses.

Results: Median duration of intensive immunosuppression was 5.1 months (IQR 5.5). GVHD-related mortality rate was $36 \%$. mNGS analysis detected viral nucleotide sequences in $24 / 25$ patients. Sequences of $\geq 3$ distinct viruses were detected in 16/25 patients; Anelloviridae (24/25) and human pegivirus-1 (9/25) were the most prevalent. In 7 patients with fatal outcomes, viral sequences not assessed by routine investigations were identified with mNGS and confirmed by RT-PCR. These cases included Usutu virus (1), rubella virus (1 vaccine strain and 1 wild-type), novel human astrovirus (HAstV) MLB2 (1), classic HAstV (1), human polyomavirus 6 and 7 (2), cutavirus (1), and bufavirus (1).

(Continued on next page)
\end{abstract}

\footnotetext{
* Correspondence: marie-celine.zanella@hcuge.ch

${ }^{\dagger}$ L. Kaiser and D. L. Vu contributed equally to this work.

'Division of Infectious Diseases, Geneva University Hospitals, 4 Rue Gabrielle-Perret-Gentil, 1211, 14 Geneva, Switzerland

${ }^{2}$ Laboratory of Virology, Division of Laboratory Medicine, Geneva University Hospitals, 4 Rue Gabrielle-Perret-Gentil, 1211, 14 Geneva, Switzerland

Full list of author information is available at the end of the article
}

(C) The Author(s). 2021 Open Access This article is licensed under a Creative Commons Attribution 4.0 International License, which permits use, sharing, adaptation, distribution and reproduction in any medium or format, as long as you give appropriate credit to the original author(s) and the source, provide a link to the Creative Commons licence, and indicate if changes were made. The images or other third party material in this article are included in the article's Creative Commons licence, unless indicated otherwise in a credit line to the material. If material is not included in the article's Creative Commons licence and your intended use is not permitted by statutory regulation or exceeds the permitted use, you will need to obtain permission directly from the copyright holder. To view a copy of this licence, visit http://creativecommons.org/licenses/by/4.0/ The Creative Commons Public Domain Dedication waiver (http://creativecommons.org/publicdomain/zero/1.0/) applies to the data made available in this article, unless otherwise stated in a credit line to the data. 
(Continued from previous page)

Conclusions: Clinically unrecognized viral infections were identified in $28 \%$ of highly immunocompromised allo-HSCT recipients with steroid-refractory/dependent GvHD in consecutive samples. These identified viruses have all been previously described in humans, but have poorly understood clinical significance. Rubella virus identification raises the possibility of re-emergence from past infections or vaccinations, or re-infection.

Keywords: Viral infection, Metagenomics, Transplantation, Graft-versus-host disease, Rubella, Anellovirus, Human pegivirus, Astrovirus, Polyomavirus, Protoparvovirus

\section{Background}

Viral primary infections and reactivations are common complications after allogeneic hematopoietic stem cell transplantation (allo-HSCT) and are associated with significant morbidity and mortality $[1,2]$. Current routine clinical surveillance molecular assays detect specific nucleotide sequences targeting usual culprits including Epstein-Barr virus (EBV), cytomegalovirus (CMV), BK polyomavirus (BKPyV), and adenovirus [3]. Metagenomic next-generation sequencing's (mNGS) unbiased approach broadens viral infection diagnosis, theoretically detecting "all" viral nucleotide sequences or viral infections present [4-7], and is increasingly used in clinical investigations [8]. Allo-HSCT recipients suffering from steroid-refractory/dependent acute or chronic graft-versus-host disease (GvHD) are highly immunosuppressed patients; GvHD immune dysregulation, mucosal barrier alteration [9-13], and multiple prolonged immunosuppressive treatments create a permissive environment for opportunistic viral infections $[10,11,14]$. These clinically unrecognized viral infections can present with limited symptoms/atypical manifestations and lead to intermittent or prolonged viremia [3]. Given the nonspecific clinical features of GvHD and some viral infections, viral infections may remain clinically unrecognized due to limitations of clinical molecular assays.

We hypothesized that some viral infections, which would normally remain undiagnosed with common clinical assays, occur during intense immunosuppressive therapy in steroid-refractory/dependent GvHD. This study aimed to identify viruses that are not routinely searched by RT-PCR routine assays in clinical practice, due to the lack of knowledge. Therefore, we used mNGS on pooled plasma samples of adult allo-HSCT patients with steroidrefractory/dependent acute or chronic GvHD to look for viruses that could be missed by biased technology.

\section{Methods}

\section{Setting, study population, and design}

This observational study was conducted at the Geneva University Hospitals (HUG), Switzerland. The study protocol was approved by the Geneva Cantonal Ethics Commission (project \#2019-00511). Inclusion criteria were adult patients who received an allo-HSCT from 1 January 2016 to 31 December 2018 at the HUG, who were enrolled in the local monocentric infectious disease cohort of allo-HSCT patients, and who developed steroid-refractory/dependent acute or chronic GvHD. All included patients provided written consent before enrollment. The only exclusion criteria was the lack of informed written consent.

Plasma samples were prospectively collected during clinical management after allo-HSCT and stored in the Laboratory of Virology, HUG. We performed mNGS analysis on pooled plasma samples of each patient, collected throughout the period of intensive steroid treatment or second-line GvHD therapy.

\section{Definitions}

Steroid-refractory/dependent acute and chronic GvHD were defined according to the position statement of the GvHD experts in Schoemans et al. [15].

\section{Microbiological methods $m N G S$ and sequence analysis}

Each pool (corresponding to 4 to 10 plasma samples from each patient) was prepared to obtain a final volume of $220 \mu \mathrm{l}$. Pools were then centrifuged at $10,000 \times g$ for $10 \mathrm{~min}$ to remove cells. Two-hundred microliters of cell-free supernatant were treated with $20 \mu$ l of Turbo DNAse (2 $\mathrm{U} / \mu \mathrm{l})+24 \mu \mathrm{l}$ of $10 \mathrm{x}$ TURBO DNase Buffer (Ambion, Rotkreuz, Switzerland), according to the manufacturer's instructions. Then, the whole volume was divided into two tubes of $120 \mu \mathrm{l}$ each. One tube was then used for each of the two nucleic acid extraction procedures. Indeed, virus genome extractions were done using two previously published protocols in parallel [16], namely the RNA and DNA protocols optimized for the detection of RNA and DNA viral sequences, respectively.

For the RNA protocol, nucleic acids were extracted with TRIzol (Invitrogen, Carlsbad, CA, USA). Ribosomal RNA was removed (Ribo-Zero Gold depletion kit (Illumina, San Diego, USA) before libraries' preparation (TruSeq total RNA preparation protocol (Illumina)). Libraries' concentrations and sizes were analyzed using the Qubit (Life Technologies, Carlsbad, CA, USA) and the 2200 TapeStation instruments (Agilent, SantaClara, CA, USA), respectively. Each library was loaded individually in a single lane on the HiSeq 4000 platform (Illumina) 
using the $2 \times 100$-bp protocol with dual-indexing. The mean total number of read pairs obtained per pool was $328,936,594.84$ (range $252,670,103$ to $385,257,539$ ).

For the DNA protocol, nucleic acids were extracted with the NucliSens easyMAG magnetic bead system (bioMérieux, Geneva, Switzerland). As previously published [16], double-stranded DNA synthesis was done with the DNA polymerase I, Large Fragment (Klenow) (New England BioLabs, Ipswich, MA, USA)). Libraries were prepared using the Nextera XT (Illumina) protocol. Libraries' concentrations and sizes were analyzed using the Qubit (Life Technologies) and the 2200 TapeStation instruments (Agilent), respectively. Each library was loaded individually in a single lane on the HiSeq 4000 platform (Illumina) using the $2 \times 100$-bp protocol with dual-indexing. The mean total number of read pairs obtained per pool was 301,393,033.48 (range 122,939,325 to $377,758,795)$.

To check for potential contaminating viral sequences from environment or experimental reagents [17], four negative controls (i.e., Neg1-4) were submitted to the whole mNGS procedure. To assess the mNGS process efficiency, positive controls underwent the whole mNGS procedure (canine distemper virus (CDV)-spiked samples and a baculovirus (GenScript, Piscataway, NJ, USA) harboring 793 nucleotides of the CDV fusion gene were used as positive controls for the RNA and DNA protocols, respectively).

Paired reads were quality filtered using Trimmomatic [18]. Reads mapped against the human genome and transcriptome (hg38, gencode.V23) were removed using SNAP [19]. Remaining reads were analyzed using two methods in parallel as previously described [20]: (1) by a pipeline that used virusscan 1.0 (https://github.com/sibswiss/virusscan) to map reads against the Virosaurus database (version V90v_2018_11) (https://viralzone. expasy.org/8676), which is designed to report vertebrate viruses, and (2) by de novo assembly. Only viruses with $\geq 300$ nucleotides of coverage were reported. The raw sequence data were deposited in the NCBI Sequence Read Archive under BioProject accession number PRJNA641787.

Sequences were considered clinically recognized viruses if they corresponded to a virus known to reactivate in/be frequently found among allo-HSCT recipients [2, 4] or if the patient had a known chronic viral infection and clinically unrecognized viruses if not.

\section{Confirmatory real-time (reverse transcription-)polymerase chain reaction ( $r(R T-) P C R)$ assays}

Clinically unrecognized mNGS findings were confirmed on unpooled plasma by specific semi-quantitative or quantitative $\mathrm{r}(\mathrm{RT}-) \mathrm{PCR}$ assays as previously published; additional specimens (including plasma, cerebrospinal fluid, bronchoalveolar lavage (BAL) fluids, nasopharyngeal swabs, native urines, stools suspension, tissue biopsies or bone marrow) were tested when available and pertinent. Quantitative $r(\mathrm{RT}$-)PCR assays were done for Mamastrovirus 1 (classical) using the updated human astrovirus (HAstV) combination [21], Mamastrovirus 6 (MLB2) using the MLB2 assay [22], Usutu virus [23], and bufavirus using the BuV (NS1) assay [24]. Semiquantitative $r(\mathrm{RT}$-)PCR assays were done for cutavirus using the $\mathrm{CuV}$ (VP2) assay [24], human polyomavirus (HPyV) 6 using the VP2 assay [25], HPyV-7 using the VP2 assay [25], and rubella virus [26].

Nucleic acids from plasma, cerebrospinal fluid, BAL fluids, nasopharyngeal swabs, urine, stools resuspended in PBS, and bone marrow were extracted individually from $190 \mu \mathrm{L}$ of each specimen, spiked with $10 \mu \mathrm{L}$ of standardized CDV as internal control [27], using the NucliSENS easyMAG (bioMérieux, Geneva, Switzerland) nucleic acid kit, according to the manufacturer's instructions, and eluted in $25 \mu \mathrm{L}$. DNA and RNA were extracted from tissue biopsies using the QIAamp DNA FFPE Tissue Kit (Qiagen, Hombrechtikon, Switzerland) and High Pure FFPET RNA isolation kit (Roche Applied Sciences, Indianapolis, IN, USA), respectively, following the manufacturer's instructions. For RNA viruses, the rRT-PCR assays were performed using the one-step QuantiTect Probe RT-PCR Kit (Qiagen, Hombrechtikon, Switzerland) in a StepOne Plus instrument (Applied Biosystems, Rotkreuz, Switzerland). For DNA viruses, the rPCR assays were performed using the TaqMan Universal PCR Master Mix (Applied Biosystems) in a StepOne Plus instrument (Applied Biosystems) for cutavirus and bufavirus or in a QuantStudio 5 instrument (Applied Biosystems) for HPyV6 and 7.

For quantitative $\mathrm{r}(\mathrm{RT})$-PCR assays, standard curves and lower limit of quantifications (LOQ) were assessed using 10-fold serial dilutions of specific RNA oligonucleotides (Mamastrovirus 1 (classical) and 6 (MLB2): LOQ $=1.25 \mathrm{E} 4$ and $1.25 \mathrm{E} 3 \mathrm{RNA}$ copies $/ \mathrm{ml}$ of plasma, respectively), RNA transcript (Usutu virus: $\mathrm{LOQ}=1.32 \mathrm{E} 2 \mathrm{RNA}$ copies/ml of plasma), DNA oligonucleotides (bufavirus: $\mathrm{LOQ}=1.32 \mathrm{E} 3 \mathrm{DNA}$ copies $/ \mathrm{ml}$ of plasma), or plasmids (HPyV6 and 7: LOQ = 2.63E2 DNA copies $/ \mathrm{ml}$ of plasma each) containing the target sequences.

\section{Statistical analysis}

Categorical variables were described by counts and percentages. Continuous variables were expressed as mean and standard deviation or median and interquartile range.

\section{Results}

Patient characteristics

We identified 25 adult allo-HSCT recipients with acute or chronic steroid-refractory/dependent GvHD. Table 1 
Table 1 Patients' characteristics (25 allo-HSCT patients)

Demographics
Sex (male), $n$ (\%)
Age, median (IQR)
Allo-HSCT considered in the analysis, $\boldsymbol{n}$ (\%)
First
Second
Transplant source, $\boldsymbol{n}$ (\%)
Bone marrow
Peripheral blood cells
Underlying disease, $\boldsymbol{n}$ (\%)
Acute myeloid leukemia
Lymphoid malignancy
MDS/MDPS
Acute lymphoid leukemia
Other ${ }^{a}$
Risk score, $\boldsymbol{n}$ (\%)
Low
Intermediate
High

Donor sex, $\mathrm{M}, \boldsymbol{n}(\%)$

Donor age, median (IQR)

Donor match, $\boldsymbol{n}$ (\%)

Donor-related

CMV donor/recipient constellation, $\boldsymbol{n}$ (\%)

$+/+$

$-/+$

$+/-$

$-1-$

CMV prophylaxis, $n(\%)$

Conditioning, $\boldsymbol{n}$ (\%)

Myeloablative conditioning

\section{GvHD prophylaxis, $\boldsymbol{n}(\%)$}

Calcineurin inhibitor

Mycophenolate mofetil

Methotrexate

\section{GvHD organ, $n$ (\%)}

Digestive tract

Skin

Mouth

Liver

Lung

Eyes

Musculoskeletal
Table 1 Patients' characteristics (25 allo-HSCT patients) (Continued)

$n=25$

$16(64.0)$

$58.0(25.0)$

23 (92.0)

2 (8.0)

5 (20)

$20(80)$

$10(40.0)$

6 (24.0)

3 (12.0)

2 (8.0)

4 (16.0)

0 (0)

18 (72.0)

7 (28.0)

7 (28.0)

36 (16.5)

$10(40.0)$

12 (48.0)

1 (4.0)

7 (28.0)

$5(20.0)$

1 (4.0)

$5(20.0)$

24 (96.0)

17 (68.0)

7 (28.0)

17 (68.0)

15 (60.0)

4 (16.0)

7 (28.0)

5 (20.0)

2 (8.0)

1 (4.0)

Total

$n=25$

GvHD grade or severity, $n$ (\%)

Grade

2

9 (36.0)

3

3 (12.0)

4

$6(24.0)$

Moderate

$6(24.0)$

Severe

7 (28.0)

GvHD treatment, $\boldsymbol{n}$ (\%)

Corticosteroids 24 (96.0)

$\begin{array}{ll}\text { Ruxolitinib } & 22(88.0)\end{array}$

Calcineurin inhibitor 21 (84.0)

Mycophenolate mofetil $12(48.0)$

Photopheresis $12(48.0)$

Budenoside $\quad 5(20.0)$

Sirolimus $3(12.0)$

Tocilizumab $2(8.0)$

Other $^{\mathrm{b}} 6(24.0)$

Death, $\boldsymbol{n}$ (\%) 15 (60.0)

Median delay from allo-HSCT, months (IQR) 11.2 (15.8)

One patient could have multiple GvHD prophylaxis and treatment, multiple organs with GvHD and multiple grades of severity. CMV prophylaxis: one patient (patient Ge24) received letermovir during the period of intensive steroid treatment or second-line GvHD therapy. GvHD grade refers to acute GvHD, GvHD severity refers to chronic GvHD. In two patients, there was no information on grade/severity. Only organs with grade GvHD $\geq 2$ or severity $\geq$ moderate are reported

Abbreviations: IQR interquartile range, allo-HSCT allogeneic hematopoietic stem cell transplantation, MDS/MDPS myelodysplasic syndrome/myelodysplasic proliferative syndrome, ATG anti-thymocyte globulin

${ }^{a}$ Other includes: multiple myeloma $(n=2)$, chronic myeloid leukemia $(n=1)$ and mixed acute leukemia $(n=1)$

b Other includes: azithromycine, montelukast, prolastin, vedolisumab, nilotinib, basilixumab, ibrutinib, and/or methotrexate

shows the patient's characteristics. The median duration of intensive immunosuppression was 5.1 months (IQR 5.5 ), and $22 / 25$ patients received ruxolitinib. At the time of writing, fifteen patients have died, with 9 considered as GvHD-related.

\section{Viral sequences identified with mNGS and confirmatory analyses}

The mNGS analysis revealed viral nucleotide sequences in all patients except Ge18 (24/25). In 16/25 patients, $\geq 3$ distinct viral species were detected (Fig. 1). Figure 2 depicts the prevalence of each identified virus, and Table $\mathrm{S} 1$ provides detailed $\mathrm{mNGS}$ characteristics.

Anelloviridae (torque teno virus (TTV), torque teno minivirus (TTMV) and torque teno midivirus (TTMD 


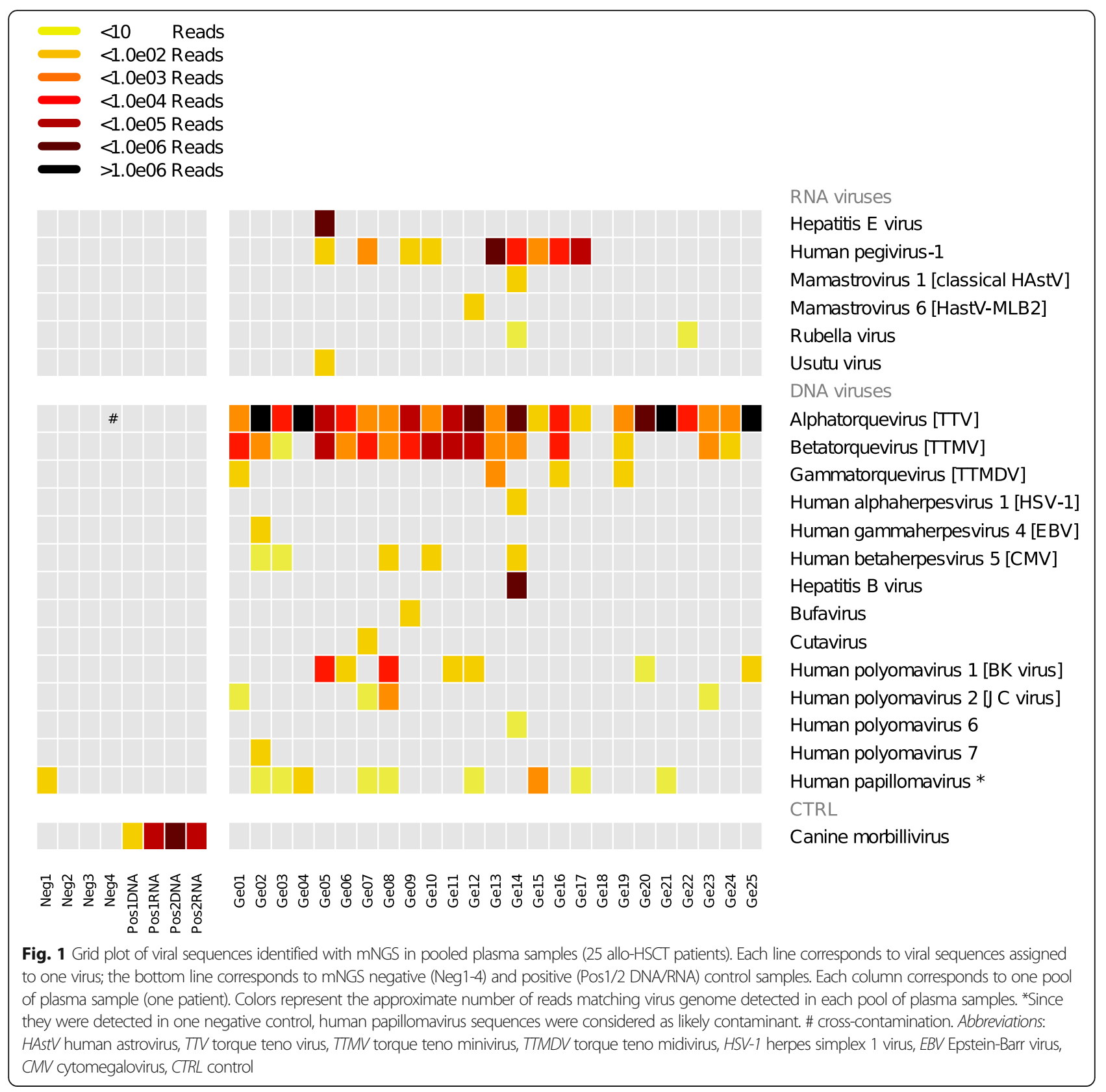

V)) and human pegivirus-1 (HPgV-1) were the most prevalent with sequences detected in $24 / 25$ and $9 / 25$ patients, respectively.

Other detected DNA viruses included BK polyomavirus (BKPyV) (7/25), CMV (5/25), and JC polyomavirus (JCPyV) (4/25), and also herpes simplex virus 1 (HSV-1), EBV, hepatitis B virus (HBV), bufavirus, cutavirus, and HPyV-6/7, each detected once (1/25). Except HPgV-1, the detected RNA viruses were less prevalent than DNA viruses and were HAstV (Mamastrovirus 1 and 6) and rubella virus, both detected twice $(2 / 25)$, and also hepatitis E (HEV) and Usutu viruses, both detected once (1/ $25)$. The de novo analysis did not reveal other relevant sequences. Interestingly, sequences for TTV (11/24), CMV (3/5), EBV (1/1), and HBV (1/1) were detected in both DNA and RNA libraries, suggesting active viral replication (Figure S1).

\section{Clinically recognized viral sequences}

Although not routinely searched in clinical practice, Anelloviridae and HPgV-1 sequences were identified in $96 \%$ and $36 \%$ of patients and were classified among clinically recognized viral sequences as they are known to be highly prevalent among immunocompromised patients. In 14/25 patients, mNGS analysis identified sequences of latent DNA viruses known to reactivate in transplant 


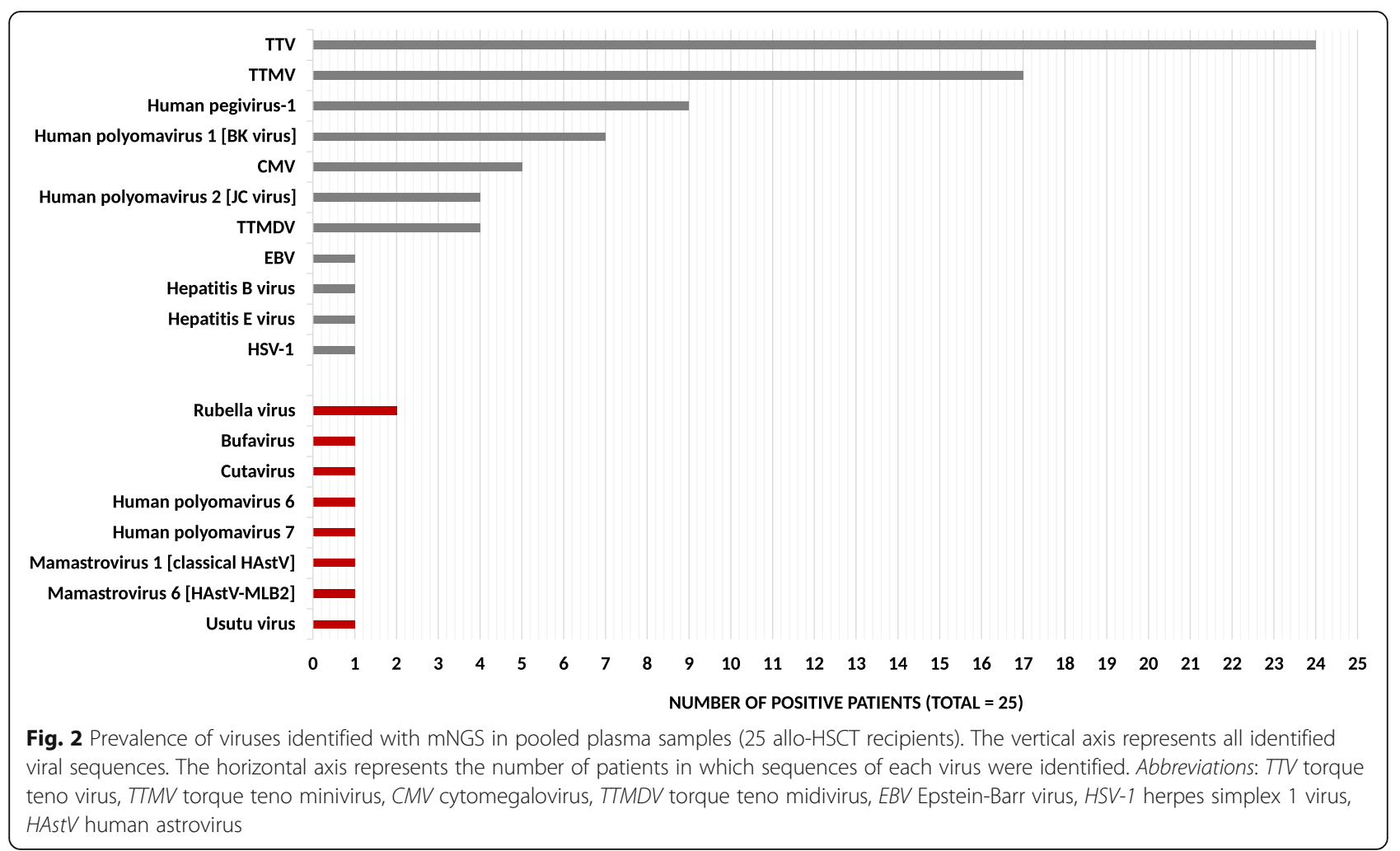

recipients (EBV, CMV, HSV-1, BKPyV, JCPyV, HBV). At the RNA level, HEV was identified once.

The $\mathrm{r}(\mathrm{RT}$-) PCR assays performed during routine investigations confirmed the mNGS analysis (Table S2): patient $\mathrm{Ge} 05$ had a chronic $\mathrm{HEV}$ infection, and patient Ge14 had chronic HBV and cutaneous HSV-1 infections, and $\mathrm{HSV}-1$ viremia. BKPyV and JCPyV were not systematically screened and were only revealed by mNGS analysis. However, BKPyV was screened in patient Ge06 and detected at low viral loads (VL) (1.41E2 and 1.44E3 copies $/ \mathrm{ml}$ ) in two plasma samples collected a few days apart from those included for mNGS analysis. In 12/25 patients, CMV and EBV were detected only by rPCR at low VL; no other expected virus was detected by routine molecular assays.

\section{Clinically unrecognized viral sequences}

We found clinically unrecognized sequences belonging to either rare and/or recently identified viruses (HAstV MLB2, Usutu virus, bufavirus, cutavirus, HPyV-6, and HPyV-7) or those not routinely assessed alongside GvHD (classic HAstV and rubella virus) in $7 / 25$ patients, whose characteristics are detailed in Table 2.

mNGS identifications of these clinically unrecognized viral sequences were confirmed by $r(\mathrm{RT}$-) PCR in unpooled plasma samples. Whenever available during/after the period of sample selection, additional specimens and/or tissue biopsies were screened over a median period of 7.1 weeks (IQR 25.3). These findings are shown in Table 3.

\section{Brief clinical description of patients harboring clinically unrecognized viral sequences \\ Ge02: HPyV-7}

A 61-year-old male, transplanted for lymphoma, developed digestive and skin GvHD; intensive immunosuppression for GvHD lasted from 12 to 16 months after transplantation (4-month sample period), when the patient died from a post-transplant EBV-related lymphoma disease. Beginning 9 months before death, we found HPyV-7 in all screened plasma samples. In addition, HPyV-7 was also found in a BAL and a bone marrow sample collected 8 months after transplantation (2.8 $\log 10$ DNA copies/ml and CT value 28 , respectively).

\section{Ge05: Usutu virus}

A 23-year-old male, transplanted for lymphoma, developed digestive skin and lung GvHD; intensive immunosuppression for GvHD lasted from 1 to 24 months after transplantation (22-month sample period), when the patient died from a disseminated fungal infection and GvHD. We found Usutu virus in 2 plasma samples collected 7 days apart, a few days after a blood transfusion, and 1.5 months before death. 
Table 2 Clinical characteristics of 7 patients with clinically unrecognized viral sequences

\begin{tabular}{llllll}
\hline $\begin{array}{l}\text { Patient's } \\
\text { code }\end{array}$ & $\begin{array}{l}\text { Age, } \\
\text { gender }\end{array}$ & $\begin{array}{l}\text { Underlying } \\
\text { disease }\end{array}$ & GvHD organ & GvHD treatments $^{\text {a }}$ & Outcome \\
\hline Ge02 & 61, M & Lymphoma & Digestive, skin & $\begin{array}{l}\text { CSA, tacrolimus, corticosteroids, sirolimus, budenoside, } \\
\text { photopheresis }\end{array}$ & $\begin{array}{l}\text { Death (GvHD- } \\
\text { related) }\end{array}$ \\
Ge05 & 23, M & Lymphoma & $\begin{array}{l}\text { Digestive, skin, } \\
\text { lung }\end{array}$ & CSA, MMF, tacrolimus, corticosteroids & Death \\
Ge07 & 60, M & ALL & Digestive & Tacrolimus, corticosteroids, photopheresis & Death \\
Ge09 & 65, M & AML & Liver, digestive ${ }^{b}$ & CSA, corticosteroids, tocilizumab & Death \\
Ge12 & 44, M & MM & Digestive, liver & CSA, corticosteroids, basiliximab, MMF & Death \\
Ge14 & $31, M$ & AML & Digestive, liver & Corticosteroids & Death \\
Ge22 & $68, M$ & MDPS & Digestive, skin & CSA, tacrolimus, corticosteroids, vedolisumab, prolastin & Death \\
\hline Ageth
\end{tabular}

Age at the time of transplantation

Abbreviations: GvHD graft-versus-host disease, $M$ male, $A L L$ acute lymphoid leukemia, $A M L$ acute myeloid leukemia, $M M$ multiple myeloma, $M D P S$ myelodysplasic proliferative syndrome, CSA cyclosporine A, MMF mycophenolate mofetil

${ }^{a}$ All patients had ruxolitinib as part of the GvHD treatment

${ }^{b}$ Digestive GvHD was not confirmed by biopsies

\section{Ge07: cutavirus}

A 60-year-old male, transplanted for acute lymphoblastic leukemia, developed digestive GvHD; intensive immunosuppression for GvHD lasted from 6 to 8 months after transplantation (2-month sample period), when the patient died from a disseminated fungal infection and GvHD. We found cutavirus in a skin biopsy performed 3 weeks before transplantation (CT of 35) and at low plasma VL for 3 months before death. Autopsy results confirmed digestive GvHD and a disseminated mold infection.

\section{Ge09: bufavirus}

A 65-year-old male, transplanted for acute myeloid leukemia (AML), developed liver GvHD; intensive immunosuppression for GvHD lasted from 5 to 7 months after transplantation ( 2 month sample period), when the patient died from GvHD. We detected bufavirus RNA in several plasma samples taken over 2 months before death. In addition, bufavirus was also found in stool samples collected the day of and 1.5 months after transplantation, at CT values of 38.2 and 34.7, respectively, and in a duodenal biopsy performed 5 months after transplantation (CT value 27.3), which revealed chronic duodenitis.

\section{Ge12: HAstV MLB2}

A 44-year-old male, transplanted for multiple myeloma, developed a digestive and liver GvHD; intensive immunosuppression for GvHD lasted from 15 days to 2 months after transplantation (2-month sample period), when the patient died from the GvHD. We detected HAstV MLB2 RNA in two plasma samples collected 14 days apart and shortly before death, in a colonic biopsy performed few days before death (CT value 27.9), and in several intestine, colonic, and bone marrow autopsy

Table 3 mNGS and r(RT-)PCR results of 7 patients with clinically unrecognized viral sequences

\begin{tabular}{|c|c|c|c|c|c|}
\hline \multirow[b]{2}{*}{$\begin{array}{l}\text { Patient's } \\
\text { code }\end{array}$} & \multirow{2}{*}{$\begin{array}{l}\text { Viral species detected } \\
\text { with mNGS } \\
\text { Virus }\end{array}$} & \multicolumn{4}{|c|}{ Viral species confirmed with $r(R T)-P C R$ assays } \\
\hline & & $\begin{array}{l}\text { Plasma, } n \\
\text { (pos/total) }\end{array}$ & $\begin{array}{l}\text { Time period } \\
\text { (days) }\end{array}$ & $\begin{array}{l}\text { Estimated viral load (mean log10 copies/ml } \\
\text { plasma or CT values) }\end{array}$ & $\begin{array}{l}\text { Other positive } \\
\text { biological specimen }\end{array}$ \\
\hline Ge02 & HPyV7 & $14 / 14$ & 279 & 4.75 & $\mathrm{BM}, \mathrm{BAL}$ \\
\hline Ge05 & Usutu virus & $2 / 5$ & 7 & 4.66 & - \\
\hline $\mathrm{Ge07}$ & Cutavirus & $6 / 10$ & 98 & 37.9 & Skin \\
\hline Ge09 & Bufavirus & $6 / 12$ & 81 & 3.73 & Stools, duodenum \\
\hline Ge12 & $\begin{array}{l}\text { Novel human astrovirus } \\
\text { MLB2 }\end{array}$ & $2 / 6$ & 14 & 3.24 & Intestine, colon, BM \\
\hline \multirow[t]{3}{*}{ Ge14 } & Rubella virus & $3 / 5$ & 39 & 37.9 & - \\
\hline & Classic human astrovirus & $5 / 11$ & 25 & 5.56 & - \\
\hline & HPyV6 & $11 / 11$ & 74 & 3.76 & - \\
\hline Ge22 & Rubella virus & $2 / 8$ & 3 & 36.7 & - \\
\hline
\end{tabular}

Pos/total corresponds to the number of positive samples on the total of screened samples. Time period corresponds to the time period during which samplings were found positive. $C T$ values above 40 were considered negative. $C T$ values are indicated in italics

Abbreviations: $B M$ bone marrow, BAL bronchoalveolar lavage, $C T$ cycle threshold 
samples (mean CT values 25.8, 27.6, 28, respectively). Autopsy results confirmed liver and digestive GvHD and revealed chronic pulmonary GvHD.

\section{Ge14: rubella virus, classic HAstV, HPyV-6}

A 31-year-old male of Chinese origin, transplanted for AML, developed digestive and liver GvHD; intensive immunosuppression for GvHD lasted 9-11 months after transplantation (3-month sample period), when the patient died from an acute intestinal perforation in the context of recurrent digestive GvHD. In several plasma samples starting 1 month before death, we found classic HAstV RNA and low VLs of a wild-type Chinese rubella strain. HPyV-6 DNA was also persistently found in plasma samples starting 2 months before death. The patient was seropositive for rubella before transplantation.

\section{Ge22: rubella virus}

A 68-year-old Portuguese male, transplanted for myelodysplastic syndrome, developed digestive and skin GvHD; intensive immunosuppression for GvHD lasted from 4 to 6 months after transplantation (6-week sample period), when the patient died from GvHD. Two plasma samples were positive for rubella-vaccine RNA at low VLs. The patient was seropositive before transplantation.

\section{Discussion}

We analyzed viral sequences by mNGS in pooled plasma samples of 25 adult allo-HSCT patients with severe steroid-refractory/dependent GvHD. Viral nucleotide sequences were found in $96 \%$ of patients, and $64 \%$ of patients had $\geq 3$ distinct viral species. Besides commensal (Anelloviridae and HPgV-1) and latent (EBV, CMV, HSV-1, BKPyV, JCPyV) viruses known to be highly prevalent/reactivate in allo-HSCT recipients, $28 \%$ of patients had clinically unrecognized viral sequences that are rarely/never reported in allo-HSCT patients, with unknown pathogenicity (bufavirus, cutavirus, HPyV-6/7, novel HAstV-MLB2, classic HAstV, rubella virus, and Usutu virus). Chronic HBV and HEV infections were also confirmed by mNGS in two patients.

Anelloviridae (TTV, TTMV, and TTMDV) were the most prevalent in this study, concordant with the high TTV viremia rate identified among allo-HSCT recipients [28-31]. The high TTV, TTMV, and TTMDV codetection rate has been previously described [30]. Chronic anelloviruses infection/re-infection is common, but disease associations remain undetermined [32]. Among allo-HSCT recipients, two studies failed to demonstrate any association between TTV viremia and immune-related complication or other viral reactivations $[28,33]$, while a third reported higher TTV viremia in patients receiving corticosteroids for GvHD [29]. A recent study found higher TTV VL at 100 days posttransplantation predicts worse overall survival, and a higher risk of acute GvHD and infections [34]. Finally, a mNGS study revealed increased detection rates and number of Anelloviridae sequences in stool samples of allo-HSCT recipients several weeks after developing digestive GvHD [5], suggesting a consequence of GvHDassociated inflammation and/or immunosuppressive therapy. Altogether, these data suggest that TTV viremia could be a potential immunosuppression-marker, requiring further investigation.

HPgV-1 (a Flaviviridae [35]) viremia occurs in 1-4\% of blood donors in developed countries [36] and up to $30 \%$ of allo-HSCT recipients but has not been associated with clinical consequences [3, 4]. Given the interaction of HPgV-1 with the immune system [37], the effect of persistent $\mathrm{HPgV}-1$ viremia requires deeper investigation.

We found four species of HPyV (JCPyV, BKPyV, HPyV-6/7), with JCPyV viremia occurring in $16 \%$ of allo-HSCT recipients, agreeing with studies where concomitant use of multiple immunosuppressive treatments was associated with increased persistent viremia risk-although progressive multifocal leucoencephalopathy was rare [38]. In another study, JCPyV DNA detection rate in plasma decreased from $4 / 22$ to $1 / 22$ patients at 3 and 12-18 months after transplantation, respectively, while viremia was not linked to any clinical manifestation [39]. Our study's BKPyV prevalence (28\%) was lower than the $54 \%$ of another study [40]. Notably, our patients did not develop hemorrhagic cystitis.

Contamination or other bioinformatics errors were excluded for each of the clinically unrecognized viral sequences, by confirming the mNGS-identified viral sequences using $\mathrm{r}(\mathrm{RT}$-)PCR on blood and non-blood samples at different time-points. These assays found cutavirus (60\%) and bufavirus (50\%), two Protoparvoviruses, in plasma samples at low VL. Interestingly, available skin and digestive biopsy tissues, previously described as putative primary infection sites [24, 41, 42], were positive by PCR at or shortly before transplantation, indicating viral latency and reactivation under immunosuppressive conditions. Bufavirus was first discovered in stool samples of children in 2012, and the stool prevalence is about $0.3-4.1 \%$, although high seroprevalence was identified in some countries [41, 42]. Bufavirus was recovered in stools of subjects with digestive symptoms, but not in asymptomatic controls [41]. Whether bufavirus influences syndromes attributed to digestive GvHD remains unknown. Cutavirus was discovered in 2016 in stools of diarrheic children in Brazil with a prevalence of about $1-1.6 \%$, and curiously, some associations were made with cutaneous $\mathrm{T}$ cell lymphoma [24, 41]. To our knowledge, cutavirus and bufavirus viremia have not been described before. 
Usutu virus (a Flaviviridae) is an arbovirus, endemic in Africa and several European countries, that frequently co-circulates with West Nile virus [43-45]. The virus enzootic cycle involves birds (main reservoir) and ornithophilic mosquitos (vectors); humans are incidental and dead-end hosts [43]. Less than 50 documented cases of acute Usutu virus infections have been reported in humans, most of them corresponding to the identification of Usutu virus genome in donated blood samples [45]. Human Usutu virus infection can be asymptomatic or associated with various clinical manifestations, including fever, rash, and neurological manifestations; the virus genome was detected in some cases in CSF or blood samples $[43,45,46]$. The virus can infect neurons, astrocytes, microglia cells, and induced pluripotent stem cell (IPSc)-derived human neuronal stem cells, with a reduction in cell proliferation, induction of antiviral response, and apoptosis [47]. In our study, Usutu virus was transiently identified shortly after a blood transfusion, but retrospective blood bag testing was impossible. Although blood transmission has not been described, screening over $\geq 130,000$ blood donations revealed 38 positive donors $[48,49]$. Although overt clinical consequences are absent in our patient, this flavivirus is known to cause occasional complications [45] and cannot be disregarded. If transmission occurred by transfusion, it is possible that only remnant RNA was transmitted, not infectious virus.

Astroviruses are well-recognized enteric viruses infecting mainly children, elderly, and immunocompromised patients [50]. HAstV MLB2 was identified a decade ago [51], and since then, it has been demonstrated that it is circulating in every continent [50]. In addition, it is associated with systemic and central nervous system infections $[7,52]$ and has been identified in stool samples of asymptomatic children [53]. In our mNGS study, classic HAstV was found in plasma samples of a patient with digestive GvHD shortly before death, and HAstV MLB2 was found at low VL in plasma samples of a patient with diarrhea due to digestive GvHD. In the latter, autopsy confirmed the presence of HAstV MLB2 RNA in several intestinal and colonic samples at significant VLs. The presence of astrovirus MLB2 in the digestive tract of the patient is thus evident, and HAstV viremia plausibly came from an intestinal spillover in the context of the GvHD and intestinal perforation.

HPyV-6 and 7 were discovered in 2010 [54] and have been identified in a wide range of clinical samples of healthy subjects, transplant recipients, and symptomatic immunocompetent patients [55-59]. They have been detected mostly in skin specimens of non-transplanted individuals and transplanted recipients with or without dermatological diseases, but the association with clinical manifestation is not established [55, 57, 59-61]. The reported seroprevalence rates of HPyV6 and $\mathrm{HPyV} 7$ in immunocompetent and immunocompromised adults varies from 69 to $84 \%$ and 35 to $72 \%$, respectively [3]. The transmission route, tropism, pathogenic mechanisms, and potential association with human diseases are still not established. HPyV-6 DNA prevalence in healthy blood donors is $0.1 \%$ and $0.6 \%$ in kidney transplant recipients [3], while HPyV-7 has been detected in lung transplant recipients [3], yet no obvious clinical manifestations are associated with them. We report persistent HPyV-6/7 viremia in plasma samples of $2 / 25$ allo-HSCT recipients over several months. Further investigations are needed to determine their pathogenicity.

Rubella virus was our most unexpected finding, yet the rubella reads for both patients mapped to different regions along the genome (rubella virus genome coverage of $3.69 \%$ and $5.47 \%$ for patients Ge14 and Ge22, respectively) and mNGS results were confirmed by specific rRT-PCR in several samples over a period of 39 and 3 days for patients Ge14 and Ge22, respectively.

Although rubella can persist in in vitro and animal models [62-64], it is not known to persist after vaccination or natural infection in humans, except in vaccinated immuno-deficient children [65-68]. Chronic rubella infection has also been hypothesized as causing Fuch's heterochromic iridocyclitis, although the pathophysiology remains unknown $[69,70]$. We found rubella sequences with low VLs in two patients, each with a distinct strain: a vaccine strain and a Chinese strain that was found in a patient who previously lived in China. Macrophages and keratinocytes are potential sites for rubella persistence [66], but retrospectively screened skin samples from one patient gave negative results. Both patients were seropositive before transplantation. Patients with GvHD frequently become seronegative for measles and rubella within 2 years after allo-HSCT [71]. Identification of the usual vaccine strain and a Chinese strain in a native Chinese, strongly argues for reactivation, in line with antibody loss after transplantation. Yet, we noted a decreased rubella IgG titer in one of both patients at the time of viremia, compared to the pre-transplant titers. Gonzalez et al. reported the case of a child who developed fulminant hepatitis after stem cell transplantation despite prior vaccination [72]. The hypothesis raised by the authors of a primary infection from a recently vaccinated contact implies that circulating vaccine strains in the population could be an issue for immunocompromised patients with waning antibodies. Whether rubella persistence could trigger GvHD after transplantation and where the viral reservoir would be are open questions.

A recent trial identified ruxolitinib as a second-line treatment for steroid-refractory GvHD, which has a poor prognosis and no approved clearly beneficial treatment $[14,73]$ : in the study, about $1 / 3$ patients experienced a 
grade 3 infection, highlighting the importance of monitoring patients for infections. Our study reveals that some viral infections were overlooked by standard procedures, which may indicate that the $30 \%$ of infection risk associated with ruxolitinib could be underestimated, and raises the question of including mNGS analysis in the management of high-risk patients.

Among the 7 patients with clinically unrecognized viral sequences, most sequences were identified a few weeks before and, persisted until, patients' deaths. Although neither the pathogenic nature of the viruses nor the clear associations with patient outcomes are proven, the identification of these viral sequences in patients' blood during severe GvHD is relevant and reflects the altered immune response; monitoring these infections could help adjust immunosuppressive therapies. Among these patients, with nearly daily blood sample collection, such strategies could routinely be actionable by pooling plasma samples (overcoming transient viremia problems), with the aim of excluding disseminated infections before increasing immunosuppression, and unmasking a viral infection mimicking the GvHD syndrome.

The identification of HAstV and bufavirus in digestive tracts of patients with digestive GvHD may merely reflect the patient's gut virome, but identification of enteric viruses in blood samples could indicate a disseminated infection that is triggered by GvHD inflammation or immunosuppressive treatment, which may require treatment adaptation.

These viral infections cannot be considered innocent bystanders. Most of the identified viruses can be shed asymptomatically, but certainly lead to organ disease under conditions where they become opportunistic pathogens, potentially causing unrecognized clinical features; they can also lead to a clinical exacerbation. The particular immunologic state of our population may influence this delicate balance between an indolent virus and its clinical impact.

A major limitation of this study is the small monocentric cohort. Additionally, including only allo-HSCT recipients with steroid-refractory/dependent GvHD precluded generalization of the results to all allo-HSCT recipients. Furthermore, we lacked control patients without GvHD. Despite the numerous viral infections revealed by mNGS, whether these are specific to patients with GvHD or to those treated with ruxolitinib, and if there is an association with clinical manifestations and/ or an impact on the immune state of these patients, remains to be determined by appropriate studies. Notably, according to the comparison with routine diagnostic results and confirmatory $\mathrm{r}$ (RT-)PCR, our mNGS pipeline is accurate, although with a lower sensitivity compared to specific quantitative real-time PCR assays used in most routine laboratories.

\section{Conclusions}

Blood analysis of patients with steroid-refractory/dependent GvHD revealed clinically unrecognized viral sequences in $28 \%$ of patients, including rubella virus, novel protoparvoviruses, HPyV-6/7, Usutu virus, and HAstV-MLB2. These viruses have been described in humans, but rarely reported as causes of disease in allo-HSCT patients, or have unknown pathogenicity. Rubella virus identifications imply possible reemergence from past infection or vaccination. Further investigations are needed to understand the clinical significance of these infections.

\section{Supplementary Information}

The online version contains supplementary material available at https://doi. org/10.1186/s40168-020-00953-3.

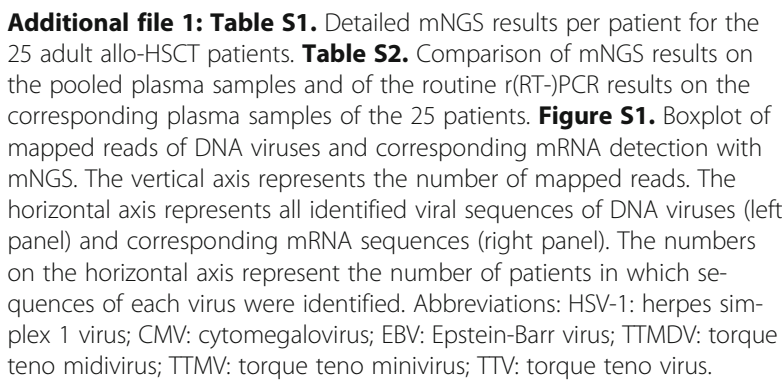

Additional file 1: Table S1. Detailed $\mathrm{mNGS}$ results per patient for the 25 adult allo-HSCT patients. Table S2. Comparison of mNGS results on the pooled plasma samples and of the routine r(RT-)PCR results on the corresponding plasma samples of the 25 patients. Figure S1. Boxplot of mapped reads of DNA viruses and corresponding mRNA detection with mNGS. The vertical axis represents the number of mapped reads. The horizontal axis represents all identified viral sequences of DNA viruses (left panel) and corresponding mRNA sequences (right panel). The numbers on the horizontal axis represent the number of patients in which sequences of each virus were identified. Abbreviations: HSV-1: herpes simplex 1 virus; CMV: cytomegalovirus; EBV: Epstein-Barr virus; TTMDV: torque teno midivirus; TTMV: torque teno minivirus; TTV: torque teno virus.

\section{Acknowledgements}

The authors would like to thank Fiona Pigny for technical assistance and Erik Boehm for editorial assistance.

\section{Authors' contributions}

$M C Z, D L V, S C$, and LK designed the research project. GV, MD, FL, and SC performed the mNGS and sequence analysis and r(RT-)PCR assays. VB, TAM, and JAL performed the biopsies histological examination. CVD, SML, and YC contributed to the interpretation of data regarding transplantation. MCZ, DLV, and SC collected and interpreted the data and wrote the first draft of the manuscript. All other authors revised and commented on the manuscript. The authors read and approved the final manuscript.

\section{Funding}

This work was supported by the "Projets Recherche et Développement (PRD)" of Geneva University Hospitals (grant number PRD 17-2019-I) and the Swiss National Science Foundation (grant number 320030_179507).

\section{Availability of data and materials \\ The datasets generated and/or analyzed during the current study are available in the Dryad repository (https://doi.org/10.5061/dryad.0k6djh9xp). The raw sequence data were deposited in the NCBI Sequence Read Archive under BioProject accession number PRJNA641787.}

Ethics approval and consent to participate

The study protocol was approved by the Geneva Cantonal Ethics Commission (project \#2019-00511).

Consent for publication

Not applicable

\section{Competing interests}

The authors declare that they have no competing interests.

\section{Author details}

${ }^{1}$ Division of Infectious Diseases, Geneva University Hospitals, 4 Rue

Gabrielle-Perret-Gentil, 1211, 14 Geneva, Switzerland. 'Laboratory of Virology, 
Division of Laboratory Medicine, Geneva University Hospitals, 4 Rue Gabrielle-Perret-Gentil, 1211, 14 Geneva, Switzerland. ${ }^{3}$ University of Geneva Medical School, Geneva, Switzerland. ${ }^{4}$ iGE3 Genomics Platform, University of Geneva, Geneva, Switzerland. ${ }^{5}$ Department of Genetics and Evolution, University of Geneva, Geneva, Switzerland. ${ }^{6}$ Clinical Pathology Service, Diagnostic Department, Geneva University Hospitals, Geneva, Switzerland. ${ }^{7}$ Division of Hematology, Department of Oncology, Geneva University Hospitals, Geneva, Switzerland. ${ }^{8}$ Geneva Centre for Emerging Viral Diseases, Geneva, Switzerland.

Received: 7 November 2020 Accepted: 6 December 2020 Published online: 24 January 2021

\section{References}

1. Martin-Pena A, Aguilar-Guisado M, Espigado I, Parody R, Miguel Cisneros J. Prospective study of infectious complications in allogeneic hematopoietic stem cell transplant recipients. Clin Transpl. 2011;25:468-74.

2. Vu DL, Dayer JA, Masouridi-Levrat S, Combescure C, Boely E, Khanna N, Mueller NJ, Kleber M, Medinger M, Halter J, et al. Microbiologically documented infections after adult allogeneic hematopoietic cell transplantation: a 5-year analysis within the Swiss Transplant Cohort study. Transpl Infect Dis. 2020:e13289.

3. Zanella MCCS, Kaiser L. Beyond cytomegalovirus and Epstein-Barr virus: a review of viruses composing the blood virome of solid organ transplant and hematopoietic stem cell transplant recipients. Clin Microbiol Rev. 2020; 33:e00027-0 (In press)

4. Vu DL, Cordey S, Simonetta F, Brito F, Docquier M, Turin L, van Delden C, Boely E, Dantin C, Pradier A, et al. Human pegivirus persistence in human blood virome after allogeneic haematopoietic stem-cell transplantation. Clin Microbiol Infect. 2019;25:225-32.

5. Legoff J, Resche-Rigon M, Bouquet J, Robin M, Naccache SN, MercierDelarue S, Federman S, Samayoa E, Rousseau C, Piron P, et al. The eukaryotic gut virome in hematopoietic stem cell transplantation: new clues in enteric graft-versus-host disease. Nat Med. 2017;23:1080-5.

6. Lewandowska DW, Schreiber PW, Schuurmans MM, Ruehe B, Zagordi O, Bayard C, Greiner M, Geissberger FD, Capaul R, Zbinden A, et al. Metagenomic sequencing complements routine diagnostics in identifying viral pathogens in lung transplant recipients with unknown etiology of respiratory infection. PLoS One. 2017;12:e0177340.

7. Cordey S, Vu DL, Schibler M, L'Huillier AG, Brito F, Docquier M, Posfay-Barbe KM, Petty TJ, Turin L, Zdobnov EM, Kaiser L. Astrovirus MLB2, a new gastroenteric virus associated with meningitis and disseminated infection. Emerg Infect Dis. 2016;22:846-53.

8. Wilson MR, Sample HA, Zorn KC, Arevalo S, Yu G, Neuhaus J, Federman S, Stryke D, Briggs B, Langelier C, et al. Clinical metagenomic sequencing for diagnosis of meningitis and encephalitis. N Engl J Med. 2019;380:2327-40.

9. Matsumura-Kimoto Y, Inamoto Y, Tajima K, Kawajiri A, Tanaka T, Hirakawa T, Ino K, Asao Y, Tamogami H, Kono C, et al. Association of cumulative steroid dose with risk of infection after treatment for severe acute graft-versus-host disease. Biol Blood Marrow Transplant. 2016;22:1102-7.

10. Miller HK, Braun TM, Stillwell T, Harris AC, Choi S, Connelly J, Couriel D, Goldstein S, Kitko CL, Magenau J, et al. Infectious risk after allogeneic hematopoietic cell transplantation complicated by acute graft-versus-host disease. Biol Blood Marrow Transplant. 2017;23:522-8.

11. Srinivasan A, Wang C, Srivastava DK, Burnette K, Shenep JL, Leung W, Hayden RT. Timeline, epidemiology, and risk factors for bacterial, fungal, and viral infections in children and adolescents after allogeneic hematopoietic stem cell transplantation. Biol Blood Marrow Transplant. 2013;19:94-101.

12. Eriguchi $Y$, Takashima $S, O$ oka H, Shimoji S, Nakamura K, Uryu H, Shimoda S, Iwasaki H, Shimono N, Ayabe T, et al. Graft-versus-host disease disrupts intestinal microbial ecology by inhibiting Paneth cell production of alphadefensins. Blood. 2012;120:223-31.

13. Levinson A, Pinkney K, Jin Z, Bhatia M, Kung AL, Foca MD, George D, Garvin $J$, Sosna J, Karamehmet E, et al. Acute gastrointestinal graft-vs-host disease is associated with increased enteric bacterial bloodstream infection density in pediatric allogeneic hematopoietic cell transplant recipients. Clin Infect Dis. 2015;61:350-7.

14. Zeiser R, von Bubnoff N, Butler J, Mohty M, Niederwieser D, Or R, Szer J, Wagner EM, Zuckerman T, Mahuzier B, et al. Ruxolitinib for glucocorticoidrefractory acute graft-versus-host disease. N Engl J Med. 2020;382:1800-10.
15. Schoemans HM, Lee SJ, Ferrara JL, Wolff D, Levine JE, Schultz KR, Shaw BE, Flowers ME, Ruutu T, Greinix H, et al. EBMT-NIH-CIBMTR Task Force position statement on standardized terminology \& guidance for graft-versus-host disease assessment. Bone Marrow Transplant. 2018;53:1401-15.

16. Petty TJ, Cordey S, Padioleau I, Docquier M, Turin L, Preynat-Seauve O, Zdobnov EM, Kaiser L. Comprehensive human virus screening using highthroughput sequencing with a user-friendly representation of bioinformatics analysis: a pilot study. J Clin Microbiol. 2014;52:3351-61.

17. Asplund M, Kjartansdottir KR, Mollerup S, Vinner L, Fridholm H, Herrera JAR, Friis-Nielsen J, Hansen TA, Jensen RH, Nielsen IB, et al. Contaminating viral sequences in high-throughput sequencing viromics: a linkage study of 700 sequencing libraries. Clin Microbiol Infect. 2019;25:1277-85.

18. Bolger AM, Lohse M, Usadel B. Trimmomatic: a flexible trimmer for Illumina sequence data. Bioinformatics. 2014;30:2114-20.

19. Naccache SN, Federman S, Veeraraghavan N, Zaharia M, Lee D, Samayoa E, Bouquet J, Greninger AL, Luk KC, Enge B, et al. A cloud-compatible bioinformatics pipeline for ultrarapid pathogen identification from nextgeneration sequencing of clinical samples. Genome Res. 2014;24:1180-92.

20. Fernandes JF, Laubscher F, Held J, Eckerle I, Docquier M, Grobusch MP, Mordmuller B, Kaiser L, Cordey S. Unbiased metagenomic next-generation sequencing of blood from hospitalized febrile children in Gabon. Emerg Microbes Infect. 2020;9:1242-4.

21. Cordey S, Vu DL, Zanella MC, Turin L, Mamin A, Kaiser L. Novel and classical human astroviruses in stool and cerebrospinal fluid: comprehensive screening in a tertiary care hospital, Switzerland. Emerg Microbes Infect. 2017;6:e84.

22. Holtz LR, Wylie KM, Sodergren E, Jiang Y, Franz CJ, Weinstock GM, Storch GA, Wang D. Astrovirus MLB2 viremia in febrile child. Emerg Infect Dis. 2011; 17:2050-2.

23. Nikolay B, Weidmann M, Dupressoir A, Faye O, Boye CS, Diallo M, Sall AA. Development of a Usutu virus specific real-time reverse transcription PCR assay based on sequenced strains from Africa and Europe. J Virol Methods. 2014;197:51-4.

24. Vaisanen E, Fu Y, Koskenmies S, Fyhrquist N, Wang Y, Keinonen A, Makisalo H, Vakeva L, Pitkanen S, Ranki A, et al. Cutavirus DNA in malignant and nonmalignant skin of cutaneous T-cell lymphoma and organ transplant patients but not of healthy adults. Clin Infect Dis. 2019;68:1904-10.

25. Antonsson A, Bialasiewicz S, Rockett RJ, Jacob K, Bennett IC, Sloots TP. Exploring the prevalence of ten polyomaviruses and two herpes viruses in breast cancer. PLoS One. 2012;7:e39842.

26. Claus C, Bergs S, Emmrich NC, Hubschen JM, Mankertz A, Liebert UG. A sensitive one-step TaqMan amplification approach for detection of rubella virus clade I and II genotypes in clinical samples. Arch Virol. 2017;162:477-86.

27. Schibler M, Yerly S, Vieille G, Docquier M, Turin L, Kaiser L, Tapparel C. Critical analysis of rhinovirus RNA load quantification by real-time reverse transcription-PCR. J Clin Microbiol. 2012;50:2868-72.

28. Wohlfarth P, Leiner M, Schoergenhofer C, Hopfinger G, Goerzer I, Puchhammer-Stoeckl E, Rabitsch W. Torquetenovirus dynamics and immune marker properties in patients following allogeneic hematopoietic stem cell transplantation: a prospective longitudinal study. Biol Blood Marrow Transplant. 2018;24:194-9.

29. Albert E, Solano C, Gimenez E, Focosi D, Perez A, Macera L, Pinana UL, Mateo EM, Boluda JCH, Maggi F, Navarro D. Kinetics of Alphatorquevirus plasma DNAemia at late times after allogeneic hematopoietic stem cell transplantation. Med Microbiol Immunol. 2019;208:253-8.

30. DL. V. Pegivirus in hematopoietic stem cell transplant recipients: a longitudinal prevalence study and association with immune reconstitution and clinical outcomes. In: EBMT; 2017.

31. Gilles R, Herling M, Holtick U, Heger E, Awerkiew S, Fish I, Holler K, Sierra S, Knops E, Kaiser R, et al. Dynamics of Torque Teno virus viremia could predict risk of complications after allogeneic hematopoietic stem cell transplantation. Med Microbiol Immunol. 2017;206:355-62.

32. Kaczorowska J, van der Hoek L. Human Anelloviruses: diverse, omnipresent and commensal members of the virome. FEMS Microbiol Rev. 2020.

33. Schmitz J, Kobbe G, Kondakci M, Schuler E, Magorsch M, Adams O. The value of Torque Teno Virus (TTV) as a marker for the degree of immunosuppression in adult patients after hematopoietic stem cell transplantation (HSCT). Biol Blood Marrow Transplant. 2019.

34. Pradier A, Masouridi-Levrat S, Bosshard C, Dantin C, Vu DL, Zanella MC, Boely E, Tapparel C, Kaiser L, Chalandon Y, et al. Torque Teno Virus as a 
potential biomarker for complications and survival after allogeneic hematopoietic stem cell transplantation. Front Immunol. 2020;11:998.

35. 10th report of the International Committee on Taxonomy of Viruses (ICTV). 2018.https://talk.ictvonline.org/ictv-reports/ictv_online_report/.

36. Yang N, Dai R, Zhang X. Global prevalence of human pegivirus-1 in healthy volunteer blood donors: a systematic review and meta-analysis. Vox Sang. 2020;115:107-19.

37. Stapleton JT, Chaloner K, Martenson JA, Zhang J, Klinzman D, Xiang J, Sauter W, Desai SN, Landay A. GB virus C infection is associated with altered lymphocyte subset distribution and reduced T cell activation and proliferation in HIV-infected individuals. PLoS One. 2012;7:e50563.

38. Wittmann T, Horowitz N, Benyamini N, Henig I, Zuckerman T, Rowe JM, KraOz Z, Szwarcwort Cohen M, Oren I, Avivi I. JC polyomavirus reactivation is common following allogeneic stem cell transplantation and its preemptive detection may prevent lethal complications. Bone Marrow Transplant. 2015; 50:984-91.

39. Tan CS, Broge TA Jr, Ngo L, Gheuens S, Viscidi R, Bord E, Rosenblatt J, Wong M, Avigan D, Koralnik IJ. Immune reconstitution after allogeneic hematopoietic stem cell transplantation is associated with selective control of JC virus reactivation. Biol Blood Marrow Transplant. 2014;20:992-9.

40. Hill JA, Mayer BT, Xie H, Leisenring WM, Huang ML, Stevens-Ayers T, Milano F, Delaney C, Sorror ML, Sandmaier BM, et al. The cumulative burden of double-stranded DNA virus detection after allogeneic HCT is associated with increased mortality. Blood. 2017.

41. Vaisanen E, Fu Y, Hedman K, Soderlund-Venermo M. Human protoparvoviruses. Viruses. 2017;9.

42. Vaisanen E, Paloniemi M, Kuisma I, Lithovius V, Kumar A, Franssila R, Ahmed K, Delwart E, Vesikari T, Hedman K, Soderlund-Venermo M. Epidemiology of two human protoparvoviruses, bufavirus and tusavirus. Sci Rep. 2016;6: 39267.

43. Gaibani P, Rossini G. An overview of Usutu virus. Microbes Infect. 2017;19: 382-7.

44. Nikolay B. A review of West Nile and Usutu virus co-circulation in Europe: how much do transmission cycles overlap? Trans R Soc Trop Med Hyg. 2015;109:609-18

45. Cle M, Beck C, Salinas S, Lecollinet S, Gutierrez S, Van de Perre P, Baldet T, Foulongne V, Simonin Y. Usutu virus: a new threat? Epidemiol Infect. 2019; 147:e232.

46. Simonin Y, Sillam O, Carles MJ, Gutierrez S, Gil P, Constant O, Martin MF, Girard G, Van de Perre P, Salinas S, et al. Human Usutu virus infection with atypical neurologic presentation, Montpellier, France, 2016. Emerg Infect Dis. 2018;24:875-8.

47. Salinas S, Constant O, Desmetz C, Barthelemy J, Lemaitre JM, Milhavet O, Nagot N, Foulongne V, Perrin FE, Saiz JC, et al. Deleterious effect of Usutu virus on human neural cells. PLoS Negl Trop Dis. 2017;11:e0005913.

48. Domanovic D, Gossner CM, Lieshout-Krikke R, Mayr W, Baroti-Toth K, Dobrota AM, Escoval MA, Henseler O, Jungbauer C, Liumbruno G, et al. West Nile and Usutu virus infections and challenges to blood safety in the European Union. Emerg Infect Dis. 2019;25:1050-7.

49. Zaaijer HL, Slot E, Molier M, Reusken C, Koppelman M. Usutu virus infection in Dutch blood donors. Transfusion. 2019;59:2931-7.

50. Vu DL, Bosch A, Pinto RM, Guix S. Epidemiology of classic and novel human astrovirus: gastroenteritis and beyond. Viruses. 2017:9.

51. Finkbeiner SR, Holtz LR, Jiang Y, Rajendran P, Franz CJ, Zhao G, Kang G, Wang D. Human stool contains a previously unrecognized diversity of novel astroviruses. Virol J. 2009;6:161.

52. Cordey S, Hartley MA, Keitel K, Laubscher F, Brito F, Junier T, Kagoro F, Samaka J, Masimba J, Said Z, et al. Detection of novel astroviruses MLB1 and MLB2 in the sera of febrile Tanzanian children. Emerg Microbes Infect. 2018;7:27.

53. Vu DL, Sabria A, Aregall N, Michl K, Sabria J, Rodriguez Garrido V, Goterris L, Bosch A, Pinto RM, Guix S. A Spanish case-control study in < 5 year-old children reveals the lack of association between MLB and VA astrovirus and diarrhea. Sci Rep. 2020;10:1760.

54. Schowalter RM, Pastrana DV, Pumphrey KA, Moyer AL, Buck CB. Merkel cell polyomavirus and two previously unknown polyomaviruses are chronically shed from human skin. Cell Host Microbe. 2010;7:509-15.

55. Ho J, Jedrych JJ, Feng H, Natalie AA, Grandinetti L, Mirvish E, Crespo MM, Yadav D, Fasanella KE, Proksell S, et al. Human polyomavirus 7-associated pruritic rash and viremia in transplant recipients. J Infect Dis. 2015;211:1560-5.
56. Bialasiewicz S, Rockett RJ, Barraclough KA, Leary D, Dudley KJ, Isbel NM, Sloots TP. Detection of recently discovered human polyomaviruses in a longitudinal kidney transplant cohort. Am J Transplant. 2016;16:2734-40.

57. Hashida Y, Higuchi T, Matsuzaki S, Nakajima K, Sano S, Daibata M. Prevalence and genetic variability of human polyomaviruses 6 and 7 in healthy skin among asymptomatic individuals. J Infect Dis. 2018;217:483-93.

58. Rockett RJ, Sloots TP, Bowes S, O'Neill N, Ye S, Robson J, Whiley DM, Lambert SB, Wang D, Nissen MD, Bialasiewicz S. Detection of novel polyomaviruses, TSPyV, HPyV6, HPyV7, HPyV9 and MWPyV in feces, urine, blood, respiratory swabs and cerebrospinal fluid. PLoS One. 2013;8:e62764.

59. Fava P, Merlino C, Novelli M, Ponti R, Galliano I, Montanari P, Tovo PA, Fierro MT, Bergallo M. HPyV6, HPyV7 and TSPyV DNA sequences detection in skin disease patients and healthy subjects. J Eur Acad Dermatol Venereol. 2016; 30:624-7.

60. Hampras SS, Locke FL, Chavez JC, Patel NS, Giuliano AR, Miller K, Gheit T, Tommasino M, Rollison DE. Prevalence of cutaneous viral infections in incident cutaneous squamous cell carcinoma detected among chronic lymphocytic leukemia and hematopoietic stem cell transplant patients. Leuk Lymphoma. 2018;59:911-7.

61. Wieland $U$, Silling $S$, Hellmich M, Potthoff A, Pfister $H$, Kreuter A. Human polyomaviruses 6, 7, 9, 10 and trichodysplasia spinulosa-associated polyomavirus in HIV-infected men. J Gen Virol. 2014;95:928-32.

62. Norval M. Mechanism of persistence of rubella virus in LLC-MK2 cells. J Gen Virol. 1979;43:289-98.

63. Williams MP, Brawner TA, Riggs HG Jr, Roehrig JT. Characteristics of a persistent rubella infection in a human cell line. J Gen Virol. 1981;52:321-8.

64. Oxford JS, Potter CW. Persistent rubella virus infection in laboratory animals. Arch Gesamte Virusforsch. 1971;34:75-81.

65. Neven B, Perot P, Bruneau J, Pasquet M, Ramirez M, Diana JS, Luzi S, CorreCatelin N, Chardot C, Moshous D, et al. Cutaneous and visceral chronic granulomatous disease triggered by a rubella virus vaccine strain in children with primary immunodeficiencies. Clin Infect Dis. 2017;64:83-6.

66. Perelygina L, Plotkin S, Russo P, Hautala T, Bonilla F, Ochs HD, Joshi A, Routes J, Patel K, Wehr C, et al. Rubella persistence in epidermal keratinocytes and granuloma M2 macrophages in patients with primary immunodeficiencies. J Allergy Clin Immunol. 2016;138:1436-1439 e1411.

67. Buchbinder D, Hauck F, Albert MH, Rack A, Bakhtiar S, Shcherbina A, Deripapa E, Sullivan KE, Perelygina L, Eloit M, et al. Rubella virus-associated cutaneous granulomatous disease: a unique complication in immunedeficient patients, not limited to DNA repair disorders. J Clin Immunol. 2019; 39:81-9.

68. Bodemer C, Sauvage V, Mahlaoui N, Cheval J, Couderc T, Leclerc-Mercier S, Debre M, Pellier I, Gagnieur L, Fraitag S, et al. Live rubella virus vaccine longterm persistence as an antigenic trigger of cutaneous granulomas in patients with primary immunodeficiency. Clin Microbiol Infect. 2014;20: O656-63.

69. Liu Y, Takusagawa HL, Chen TC, Pasquale LR. Fuchs heterochromic iridocyclitis and the rubella virus. Int Ophthalmol Clin. 2011;51:1-12.

70. Gonzales JA, Hinterwirth A, Shantha J, Wang K, Zhong L, Cummings SL, Qian Y, Wilson MR, Acharya NR, Doan T. Association of ocular inflammation and rubella virus persistence. JAMA Ophthalmol. 2019;137:435-8.

71. Kawamura K, Yamazaki R, Akahoshi Y, Nakano H, Ugai T, Wada H, Yamasaki R, Ishihara Y, Sakamoto K, Ashizawa M, et al. Evaluation of the immune status against measles, mumps, and rubella in adult allogeneic hematopoietic stem cell transplantation recipients. Hematology. 2015;20:77-82.

72. Gonzalez Vicent M, Molina Angulo B, Echevarria Mayo JE, Diaz Perez MA. Vaccine rubella: a rare cause of post-transplant hematopoietic death, but a major public health problem. Open Forum Infect Dis. 2018;5:ofy235.

73. Chao N. Finally, a successful randomized trial for GVHD. N Engl J Med. 2020; 382:1853-4.

\section{Publisher's Note}

Springer Nature remains neutral with regard to jurisdictional claims in published maps and institutional affiliations. 